\title{
A fast partial parse of natural language sentences using a connectionist method
}

\author{
Caroline Lyon \\ Division of Computer Science \\ University of Hertfordshire \\ Hatfield AL10 9AB, UK \\ comrcml@herts.ac.uk
}

\author{
Bob Dickerson \\ Division of Computer Science \\ University of Hertfordshire \\ Hatfield AL10 9AB, UK \\ comqrgd@herts.ac.uk
}

\begin{abstract}
The pattern matching capabilities of neural networks can be used to locate syntactic constituents of natural language. This paper describes a fully automated hybrid system, using neural nets operating within a grammatic framework. It addresses the representation of language for connectionist processing, and describes methods of constraining the problem size. The function of the network is briefly explained, and results are given.
\end{abstract}

\section{Introduction}

The pattern matching capabilities of neural networks can be used to detect syntactic constituents of natural language. This approach bears comparison with probabilistic systems, but has the advantage that negative as well as positive information can be modelled. Also, most computation is done in advance, when the nets are trained, so the run time computational load is low. In this work neural networks are used as part of a fully automated system that finds a partial parse of declarative sentences. The connectionist processors operate within a grammatic framework, and are supported by pre-processors that filter the data and reduce the problem to a computationally tractable size. A prototype can be accessed via the Internet, on which users can try their own text (details from the authors). It will take a sentence, locate the subject and then find the head of the subject. Typically 10 sentences take about 2 seconds, 50 sentences about 4 seconds, to process on a Sparc10 workstation. Using the prototype on technical manuals the subject and its head can be detected in over $90 \%$ of cases (See Section 7).

The well known complexity of parsing is addressed by decomposing the problem, and then locating one syntactic constituent at a time. The sentence is first decomposed into the broad syntactic categories

$$
\text { pre-subject - subject - predicate }
$$

by locating the subject. Then these constituents can be processed further. The underlying principle employed at each step is to take a sentence, or part of a sentence, and generate strings with the boundary markers of the syntactic constituent in question placed in all possible positions. Then a neural net selects the string with the correct placement.

This paper gives an overview of how natural language is converted to a representation that the neural nets can handle, and how the problem is reduced to a manageable size. It then outlines the neural net selection process. A comprehensive account is given in Lyon (1994); descriptions of the neural net process are also in Lyon (1993) and Lyon and Frank (1992). This is a hybrid system. The core process is data driven, as the parameters of the neural networks are derived from training text. The neural net is trained in supervised mode on examples that have been manually marked "correct" and "incorrect". It will then be able to classify unseen examples. However, the initial processing stages, in which the problem size is constrained, operate within a skeletal grammatic framework. Computational tractability is further addressed by reducing data through the application of prohibitive rules as local constraints. The pruning process is remarkably effective.

\section{The corpus of sentences from technical manuals}

This work has principally been developed on text of technical manuals from Perkins Engines Ltd., which have been translated by a semi-automatic process (Pym, 1993). Now, a partial parse can support such a process. For instance, frequently occurring modal verbs such as "must" are not dis- 

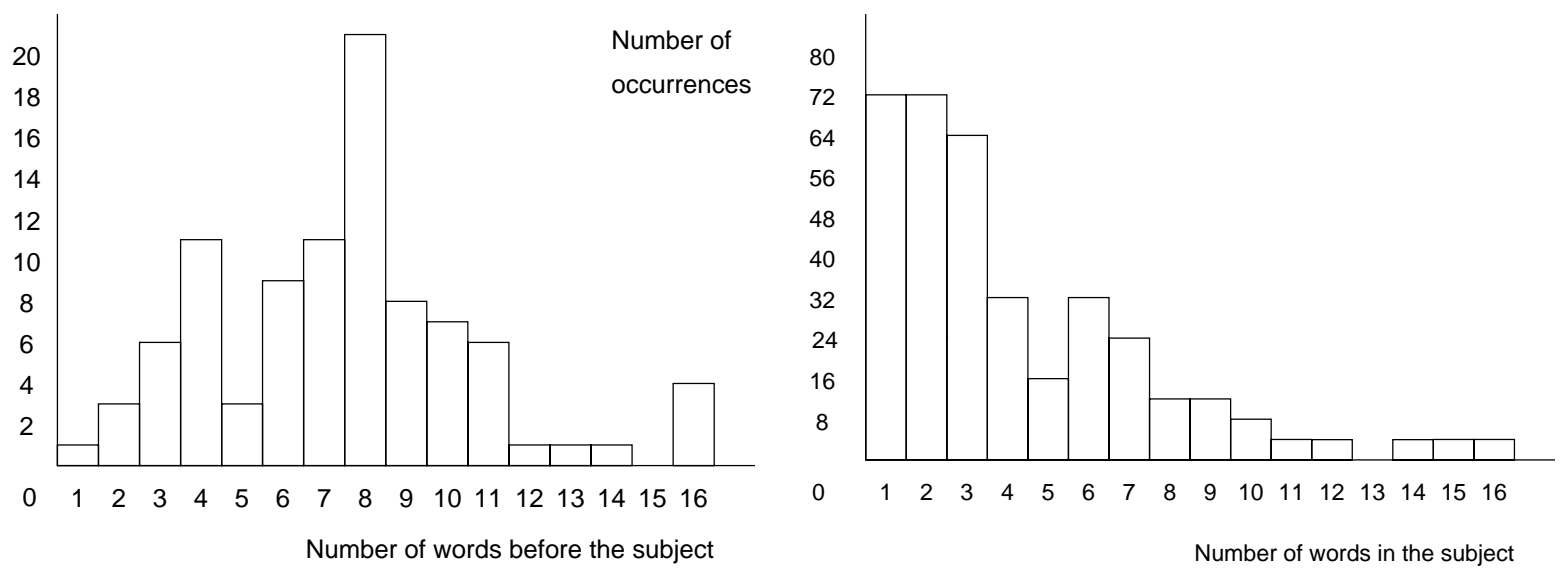

Figure 1: The frequency of constituent length for pre-subject and subject in 351 sentences

tinguished by number in English, but they are in many other languages. It is necessary to locate the subject, then identify the head and determine its number in order to translate the main verb correctly in sentences like (1) below.

If a cooler is fitted to the gearbox, [ the pipe [ connections ] of the cooler ] must be regularly checked for corrosion.

This parser has been trained to find the syntactic subject head that agrees in number with the main verb. The manuals are written using the PACE (Perkins Approved Clear English) guidelines, with the aim of producing clear, unambiguous texts. All declarative sentences have been extracted for processing: about half were imperatives. This level of classification can be done automatically in future. Table 1 and Figure 1 show some of the characteristics of the corpus.

\begin{tabular}{|l|c|}
\hline Number of sentences & 351 \\
\hline Average length & 17.98 words \\
\hline No. of subordinate clauses & \\
In pre-subject & 65 \\
In subject & 19 \\
In predicate & 136 \\
\hline Co-ordinated clauses & 50 \\
\hline
\end{tabular}

Punctuation marks are counted as words, formulae as 1 word.

Table 1: Corpus statistics

\section{$3 \quad$ Language representation (I)}

In order to reconcile computational feasibility to empirical realism an appropriate form of language representation is critical. The first step in constraining the problem size is to partition an unlimited vocabulary into a restricted number of partof-speech tags. Different stages of processing place different requirements on the classification system, so customised tagsets have been developed. For the first processing stage we need to place the subject markers, and, as a further task, disambiguate tags. It was not found necessary to use number information at this stage. For example, consider the sentence:

Still waters run deep. (2)

The word "waters" could be a 3rd person, singular, present verb or a plural noun. However, in order to disambiguate the tag and place the subject markers it is only necessary to know that it is a noun or else a verb. The sentence parsed at the first level returns:

[ Still waters] run deep.

The tagset used at this stage, mode 1 , has 21 classes, not distinguished for number. However, the head of the subject is then found and number agreement with the verb can be assessed. At this stage the tagset, mode 2, includes number information and has 28 classes. Devising optimal tagsets for given tasks is a field in which further work is planned. We need larger tagsets to capture more linguistic information, but smaller ones to constrain the computational load. Information theoretic tools can be used to find the entropy of different tag sequence languages, and support decisions on representation.

A functional approach is taken to tagging: words are allocated to classes depending on their syntactic role. For instance, superlative adjectives can act as nouns, so they are initially given the 2 tags: noun or adjective. This approach can be extended by taking adjacent words which act jointly as single lexical items as a unit. Thus the pair 
"most < adjective $>$ " is taken as a single superlative adjective.

Text is automatically tagged using the first modules of the CLAWS program (1985 version), in which words are allocated one or more tags from 134 classes (Garside, 1987). These 134 tags are then mapped onto the small customised tagsets. Tag disambiguation is part of the parsing task, handled by the neural net and its pre-processor. This version of CLAWS has a dictionary of about 6,300 words only. Other words are tagged using suffix information, or else defaults are invoked. The correct tag is almost always included in the set allocated, but more tags than necessary are often proposed. A larger dictionary in later versions will address this problem.

\section{Representing syntactic boundary markers}

In the same way that tags are allocated to words, or to punctuation marks, they can represent the boundaries of syntactic constituents, such as noun phrases and verb phrases. Boundary markers can be considered invisible tags, or hypertags, which have probabilistic relationships with adjacent tags in the same way that words do. Atwell (1987) and Church (1989) have used this approach. If embedded syntactic constituents are sought in a single pass, this can lead to computational overload (Pocock and Atwell, 1994). Our approach uses a similar concept, but differs in that embedded syntactic constituents are detected one at a time in separate steps. There are only 2 hypertags - the opening and closing brackets marking the possible location(s) of the syntactic constituent in question. Using this representation a hierarchical language structure is converted to a string of tags represented by a linear vector.

\section{Constraining the generation of candidate strings}

This system generates sets of tag strings for each sentence, with the hypertags placed in all possible positions. Thus, for the subject detection task:

Then the performance of the pump must be monitored.

will generate strings of tags including:

[ Then ] the performance of the pump must be monitored.

[ Then the ] performance of the pump must be monitored.

Then [ the performance of the ] pump must be monitored. (3.n)

Then [ the performance of the pump ] must be monitored. $\quad(3 . n+1)$
Hypertags are always inserted in pairs, so that closure is enforced. There were arbitrary limits of a maximum of 10 words in the pre-subject and 10 words within the subject for the initial work described here. These are now extended to 15 words in the pre-subject, 12 in the subject - see Section 7. There must be at least one word beyond the end of the subject and before the end-of-sentence mark. Therefore, using the initial restrictions, in a sentence of 22 words or more (counting punctuation marks as words) there could be 100 alternative placements. However, some words will have more than one possible tag. For instance, in sentence (1) above 5 words have 2 alternative tags, which will generate $2^{5}$ possible strings before the hypertags are inserted. Since there are 22 words (including punctuation ) the total number of strings would be $2^{5} * 100=3200$. It is not feasible to detect one string out of this number: if the classifier marked all strings incorrect the percentage wrongly classified would only be $0.03 \%$, yet it would be quite useless. In order to find the correct string most of the outside candidates must be dropped,

\section{The skeletal grammatic framework}

A minimal grammar, set out in Lyon (1994) in EBNF form, is composed of 9 rules. For instance, the subject must contain a noun-type word. Applying this particular rule to sentence (3) above would eliminate candidate strings (3.1) and (3.2). We also have the 2 arbitrary limits on length of pre-subject and subject. There is a small set of 4 extensions to the grammar, or semi-local constraints. For instance, if a relative pronoun occurs, then a verb must follow in that constituent. On the technical manuals the constraints of the grammatic framework put up to $6 \%$ of declarative sentences outside our system, most commonly because the pre-subject is too long. A small number are excluded because the system cannot handle a co-ordinated head. With the length of pre-subject extended to 15 words, and subject to 12 words, an average of $2 \%$ are excluded ( 7 out of 351 ).

\section{Prohibition tables}

The grammatic framework alone does not reduce the number of candidate strings sufficiently for the subject detection stage. This problem is addressed further by a method suggested by Barton, Berwick and Ristad (1987) that local constraints can rein in the generation of an intractable number of possibilities. In our system the local constraints are prohibited tag pairs and triples. These are adjacent tags which are not allowed, such as "determiner - verb" or 


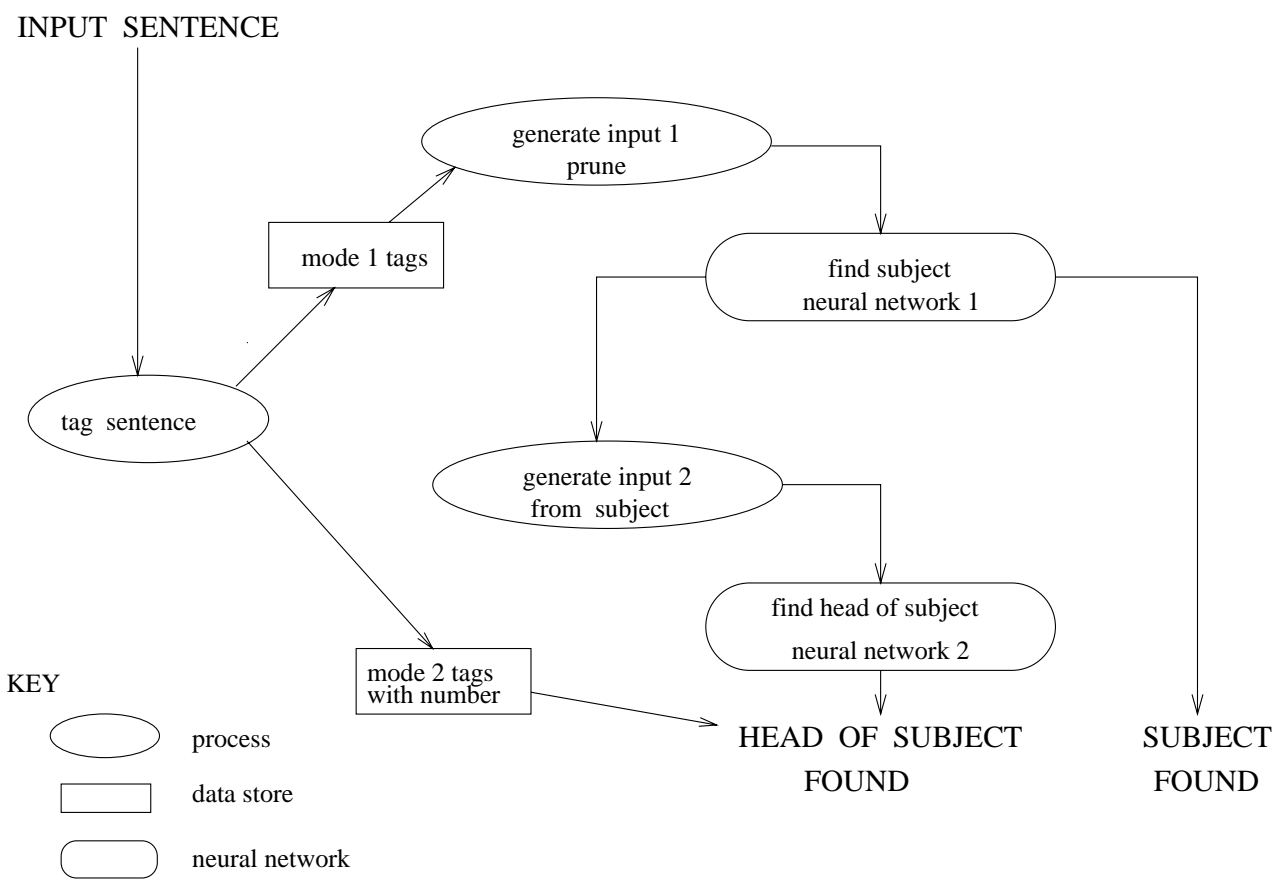

Figure 2: Overview of the syntactic pattern recognition process

"start of subject - verb". If during the generation of a candidate string a prohibited tuple is encountered, then the process is aborted. There are about 100 prohibited pairs and 120 triples. By using these methods the number of candidate strings is drastically reduced. For the technical manuals an average of 4 strings, seldom more than 15 strings, are left. Around $25 \%$ of sentences are left with a single string. These filters or "rules" differ fundamentally from generative rules that produce allowable strings in a language. In those cases only productions that are explicitly admitted are allowed. Here, in contrast, anything that is not expressly prohibited is allowed. At this stage the data is ready to present to the neural net. Figure 2 gives an overview of the whole process.

\section{Language representation (II)}

Different network architectures have been investigated, but they all share the same input and output representation. The output from the net is a vector whose 2 elements, or nodes, represent "correct" and "incorrect", "yes" and "no" - see Figure 3 . The input to the net is derived from the candidate strings, the sequences of tags and hypertags. These must be converted to binary vectors. Each element of the vector will represent a feature that is flagged 0 or 1 , absent or present.

Though the form in which the vector is written may give an illusion of representing order, no se- quential order is maintained. A method of representing a sequence must be chosen. The sequential order of the input is captured here, partially, by taking adjacent tags, pairs and triples, as the feature elements. The individual tags are converted to a bipos and tripos representation. Using this method each tag is in 3 tripos and 2 bipos elements. This highly redundant code will aid the processing of sparse data typical of natural language.

For most of the work described here the sentence was dynamically truncated 2 words beyond the hypertag marking the close of the subject. This process has now been improved by going further along the sentence.

\section{The function of the net}

The net that gave best results was a simple single layer net (Figure 3), derived from the Hodyne net of Wyard and Nightingale (1990). This is conventionally a "single layer" net, since there is one layer of processing nodes. Multi-layer networks, which can process linearly inseparable data, were also investigated, but are not necessary for this particular processing task. The linear separability of data is related to its order, and this system uses higher order pairs and triples as input. The question of appropriate network architecture is examined in Pao (1989), Widrow and Lehr (1992) and Lyon (1994). 


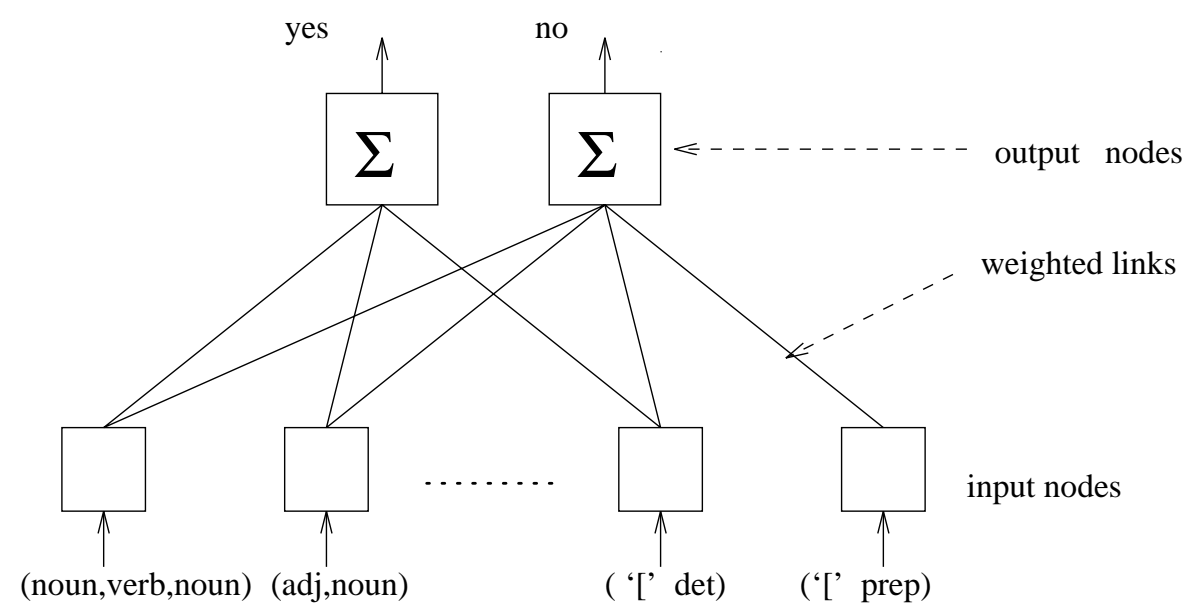

'[' represents the start of the subject. The node ('[' determiner) would occur often in both correct and incorrect strings. The node ("F' preposition) would not occur in a correct string, so it is not connected to the "yes" output node.

$\Sigma$ represents summing function.

Figure 3: The single layer net: showing the feed forward process

\section{The training process}

The net is presented with training strings whose desired classification has been manually marked. The weights on the connections between input and output nodes are adjusted until a required level of performance is reached. Then the weights are fixed and the trained net is ready to classify unseen sentences. The prototype accessible via the Internet has been trained on sentences from the technical manuals, slightly augmented.

Initially the weighted links are disabled. When a string is presented to the network in training mode, it activates a set of input nodes. If an input node is not already linked to the output node representing the desired response, it will be connected and the weight on the connection will be initialised to 1.0. Most input nodes are connected to both outputs, since most tuples occur in both grammatical and ungrammatical strings. However, some will only be connected to one output - see Figure 3.

The input layer potentially has a node for each possible tuple. With 28 tags, 2 hypertags and a start symbol the upper bound on the number of input nodes is $31^{3}+31^{2}$. In practice the maximum activated is currently about 1000 . In testing mode, if a previously unseen tuple appears it makes zero contribution to the result. The activations at the input layer are fed forward through the weighted connections to the output nodes, where they are summed. The highest output marks the winning node. If the desired node wins, then no action is taken. If the desired node does not win, then the weight on connections to the desired node are incremented, while the weights on connections to the unwanted node are decremented.

This algorithm differs from some commonly used methods. In feed forward networks trained in supervised mode to perform a classification task different penalty measures can be used to trigger a weight update. Back propagation and some single layer training methods typically minimise a metric based on the least squared error (LSE) between desired and actual activation of the output nodes. The reason why a differentiable error measure of this sort is necessary for multilayer nets is well documented, for example see Rumelhart and McClelland (1986). However, for single layer nets we can choose to update weights directly: the error at an output node can trigger weight updates on the connections that feed it. Solutions with LSE are not necessarily the same as minimising the number of misclassifications, and for certain types of data this second method of direct training may be appropriate. Now, in the natural language domain it is desirable to get information from infrequent as well as common events. Rare events, rather than being noise, can make a useful contribution to a classification task. We need a method that captures information from infrequent events, and adopt a 
direct measure of misclassification. This may be better suited to data with a "Zipfian" distribution (Shannon, 1951).

The update factor is chosen to meet several requirements. It should always be positive, and asymptotic to maximum and minimum bounds. The factor should be greatest in the central region, least as it moves away in either direction. We are currently still using the original Hodyne function because it works well in practice. The update factor is given in the following formula. If $\delta=+1$ for strengthening weights and $\delta=-1$ for weakening them, then

$$
w_{\text {new }}=\left[1+\frac{\delta * w_{\text {old }}}{1+\left(\delta * w_{\text {old }}\right)^{4}}\right] w_{\text {old }}
$$

Recall that weights are initialised to 1.0. After training we find that the weight range is bounded by

$$
10^{-3}<w<5.0
$$

Total time for training is measured in seconds. The number of iterative cycles that are necessary depends on the threshold chosen for the trained net to cross, and on details of the vector representation. The demonstration prototype takes about 15 seconds. With the most recent improved representation about 1000 strings can be trained in 1 second, to $97 \%$. The results from using these nets are given in Table 3. It was found that triples alone gave as good results as pairs and triples together. And though the nets easily train to $99 \%$ correct, the lower threshold gives slightly better generalisation and thus gives better results on the test data.

\section{The testing process}

When the trained net is run on unseen data the weights on the links are fixed. Any link that is still disabled is activated and initialised to 0 , so that tuples which have not occurred in the training corpus make no contribution to the classification task. Sentences are put through the preprocesser one at a time and the candidate strings which are generated are then presented to the network. The output is now interpreted differently. The difference between the "yes" and "no" activation levels is recorded for each string, and this score is considered a measure of grammaticality, $\Gamma$. The string with the highest $\Gamma$ score is taken as the correct one.

For the results given below, the networks were trained on part of the corpus and tested on another part of the corpus. For the prototype in which users can process their own text, the net was trained on the whole corpus, slightly augmented.

\section{Results}

There are several measures of correctness that can be taken when results are evaluated. The most lenient is whether or not the subject and head markers are placed correctly - the type of measure used in the IBM/Lancaster work (Black, Garside and Leech, 1993). Since we are working towards a hierarchical language structure, we may want the words within constituents correctly tagged, ready for the next stage of processing. "correct- A" also requires that the words within the subject are correctly tagged. The results in Tables 2 and 3 give an indication of performance levels.

\section{Using negative information}

When parses are postulated for a sentence negative as well as positive examples are likely to occur. Now, in natural language negative correlations are an important source of information: the occurrence of some words or groups of words inhibit others from following. We wish to exploit these constraints. Brill et al. (1990) recognised this, and introduced the idea of distituents. These are elements of a sentence that should be separated, as opposed to elements of constituents that cling together. Brill addresses the problem of finding a valid metric for distituency by using a generalized mutual information statistic. Distituency is marked by a mutual information minima. His method is supported by a small 4 rule grammar.

However, this approach does not fully capture the sense in which inhibitory factors play a negative and not just a neutral role. We want to distinguish between items that are unlikely to occur ever, and those that have just not happened to turn up in the training data. For example, in sentence (3) above strings $3.1,3.2$ and $3 . n$ can never be correct. These should be distinguished from possibly correct parses that are not in the training data. In order that "improbabilities" can be modelled by inhibitory connections Niles and Silverman (1990) show how a Hidden Markov Model can be implemented by a neural network.

The theoretical ground for incorporating negative examples in a language learning process originates with the work of Gold (1967), developed by Angluin (1980). He examined the process of learning the grammar of a formal language from examples. He showed that, for languages at least as high in the Chomsky hierarchy as CFGs, inference from positive data alone is strictly less powerful than inference from both positive and negative data together. To illustrate this informally consider a case of inference from a number 


\begin{tabular}{|c|c||c|c|c|}
\hline $\begin{array}{c}\text { no. of } \\
\text { training sents. }\end{array}$ & $\begin{array}{c}\text { no. of } \\
\text { test sents. }\end{array}$ & $\begin{array}{c}\text { \% sents with } \\
\text { subject } \\
\text { found }\end{array}$ & $\begin{array}{c}\text { \% sents } \\
\text { correct } \\
\text { measure A }\end{array}$ & $\begin{array}{c}\text { \% sents with } \\
\text { subject and head } \\
\text { found }\end{array}$ \\
\hline 220 & 42 & 100 & 100 & 95 \\
\hline 198 & 63 & 97 & 97 & 90 \\
\hline 204 & 58 & 95 & 95 & 93 \\
\hline 276 & 50 & 94 & & 94 \\
\hline
\end{tabular}

Table 2: Performance on text from Perkins manuals, after 6\% sentences have been excluded

\begin{tabular}{|c|c||c|c|c|}
\hline $\begin{array}{c}\text { no. of } \\
\text { training sents. }\end{array}$ & $\begin{array}{c}\text { no. of } \\
\text { test sents. }\end{array}$ & $\begin{array}{c}\text { \% sents with } \\
\text { subject } \\
\text { found }\end{array}$ & $\begin{array}{c}\text { \% sents } \\
\text { correct } \\
\text { measure A }\end{array}$ & $\begin{array}{c}\text { \% sents with } \\
\text { subject and head } \\
\text { found }\end{array}$ \\
\hline 309 & 42 & 100 & 97.6 & 97.6 \\
\hline 288 & 63 & 98.4 & 96.8 & 96.8 \\
\hline 292 & 59 & 98.3 & 98.3 & 96.6 \\
\hline 284 & 67 & 94.0 & 94.0 & 94.0 \\
\hline
\end{tabular}

Table 3: Performance on text from Perkins manuals, using improved representation and larger training set, after $2 \%$ sentences have been excluded

of examples: as they are presented to the inference machine, possible grammars are postulated. However, with positive data alone a problem of over generalization arises: the postulated grammar may be a superset of the real grammar, and sentences that are outside the real grammar could be accepted. If both positive and negative data is used, counter examples will reduce the postulated grammar so that it is nearer the real grammar. Gold developed his theory for formal languages: it is argued that similar considerations apply here. A grammar may be inferred from positive examples alone for certain subsets of regular languages (Garcia and Vidal, 1990), or an inference process may degenerate into a look up procedure if every possible positive example is stored. In these cases negative information is not required, but they are not plausible models for unbounded natural language. In our method the required parse is found by inferring the grammar from both positive and negative information, which is effectively modelled by the neural net. Future work will investigate the effect of training the networks on the positive examples alone. With our current size corpus there is not enough data.

\section{Relationship between the neural net and prohibition table}

The relationship between the neural net and the rules in the prohibition table should be seen in the following way. Any single rule prohibiting a tuple of adjacent tags could be omitted and the neural network would handle it by linking the node representing that tuple to "no" only.
However, for some processing steps we need to reduce the number of candidate tag strings presented to the neural network to manageable proportions (see Section 4). The data must be preprocessed by filtering through the prohibition rule constraints. If the number of candidate strings is within desirable bounds, such as for the head detection task, no rules are used. Our system is data driven as far as possible: the rules are invoked if they are needed to make the problem computationally tractable.

\section{Conclusion}

Our working prototype indicates that the methods described here are worth developing, and that connectionist methods can be used to generalise from the training corpus to unseen text. Since data can be represented as higher order tuples, single layer networks can be used. The traditional problems of training times do not arise. We have also used multi-layer nets on this data: they have no advantages, and perform slightly less well (Lyon, 1994).

The supporting role of the grammatic framework and the prohibition filters should not be underestimated. Whenever the scope of the system is extended it has been found necessary to enhance these elements.

The most laborious part of this work is preparing the training data. Each time the representation is modified a new set of strings is generated that need marking up. An autodidactic check is now included which speeds up this task. We run 
marked up training data through an early version of the network trained on the same data, so the results should be almost all correct. If an "incorrect" parse occurs we can then check whether that sentence was properly marked up.

Some of the features of the system described here could be used in a stochastic process. However, connectionist methods have low computational loads at runtime. Moreover, they can utilise more of the implicit information in the training data by modelling negative relationships. This is a powerful concept that can be exploited in the effort to squeeze out every available piece of useful information for natural language processing.

Future work is planned to extend this very limited partial parser, and decompose sentences further into their hierarchical constituent parts. In order to do this a number of subsidiary tasks will be addressed. The system is being improved by identifying groups of words that act as single lexical items. The decomposition of the problem can be investigated further: for instance, should the tag disambiguation task precede the placement of the subject boundary markers in a separate step? More detailed investigation of language representation issues will be undertaken. And the critical issues of investigating the most appropriate network architectures will be carried on.

\section{References}

D Angluin. 1980. Inductive inference of formal languages from positive data. Information and Control, 45.

E Atwell. 1987. Constituent-likelihood grammar. In R Garside, G Leech, and G Sampson, editors, The Computational Analysis of English: a corpus-based approach. Longman.

G E Barton, R C Berwick, and E S Ristad. 1987. Computational Complexity and Natural Language. MIT Press.

E Black, R Garside, and G Leech. 1993. Statistically driven computer grammars of English: the IBM/Lancaster approach. Rodopi.

E Brill, D Magerman, M Marcus and B Santorini. 1990. Deducing linguistic structure from the statistics of large corpora. In DARPA Speech and Natural Language Workshop.

K W Church, Bell Laboratories. 1989. A stochastic parts program and noun phrase parser for unrestricted text. In IEEE conference record of ICASSP.
P Garcia and E Vidal. 1990. Inference of ktestable languages in the strict sense and application to syntactic pattern recognition. IEEE Trans. on Pattern Analysis and Machine Intelligence, 12 .

R Garside. 1987. The CLAWS word-tagging system. In R Garside, G Leech, and G Sampson, editors, The Computational Analysis of English: a corpus based approach. Longman.

E M Gold. 1967. Language identification in the limit. Information and Control, 10.

C Lyon. 1994. The representation of natural language to enable neural networks to detect syntactic features. $\mathrm{PhD}$ thesis.

C Lyon 1993. Using neural networks to infer grammatical structures in natural language. In Proc. of IEE Colloquium on Grammatical Inference.

C Lyon and R Frank 1992. Detecting structures in natural language using a neural net with rules. In Proc. of International Conference on Artificial Neural Networks (ICANN).

L Niles and H Silverman. 1990. Combining Hidden Markov Models and Neural Network Classifiers. In IEEE conference record of ICASSP.

Yoh-Han Pao. 1989. Adaptive Pattern Recognition and Neural Networks. Addison Wesley.

R Pocock and E Atwell. 1994. Treebank trained probabilistic parsing of lattices. School of Computer Studies, Leeds University. In The SpeechOriented Probabilistic Parser Project: Final Report to MoD.

P Pym. 1993. Perkins Engines and Publications. In Proceedings of Technology and Language in Europe 2000. DGXIII-E of the European Commission.

D Rumelhart and J McClelland. 1986. Parallel Distributed Processing MIT.

C E Shannon 1951. Prediction and Entropy of Printed English. In Bell System Technical Journal.

B Widrow and M Lehr. 1992. 30 years of adaptive neural networks. In Neural networks: theoretical foundations and analysis edited by C Lau. IEEE press.

P Wyard and C Nightingale. 1990. A Single Layer Higher Order Neural Net and its Application to Context Free Grammar Recognition In Connection Science, 4. 